\title{
Cholinesterase-inhibitor Associated Mania: A Case Report and Literature Review
}

\author{
Hamza Jalal, Aravind Ganesh, Raymond Lau, John Lysack, Zahinoor Ismail
}

Can J Neurol Sci. 2014; 41: 278-280

Neuropsychiatric symptoms (NPS) are common in the middle to late stages of Alzheimer's dementia (AD), but can also present in the Mild Cognitive Impairment (MCI) phase. Since the pathophysiology of AD involves deficits in central cholinergic neurotransmission, cholinesterase inhibitors (CHEIs) are effective in treating its cognitive symptoms. Interestingly, case studies have reported the onset of mania after initiation of CHEIs in cognitively impaired patients, both with and without preexisting bipolar disorder ${ }^{1-4}$. Here we present a patient with mania as the index manifestation of a neurodegenerative disorder, who experienced recurrent mania after initiation of galantamine.

\section{Case Report}

Mrs. C is a 79-year-old woman who experienced her index manic episode in 2007, at the age of 74, presenting with euphoria, paranoia, decreased need for sleep, rapid speech, hyperactivity, impulsivity, disinhibition, and psychomotor agitation. She had no history of prior psychiatric symptoms or substance abuse, but had coronary artery disease, hypertension, a prior transient ischemic attack, atrial fibrillation, gastroesophageal reflux disease, and hypothyroidism. She was diagnosed with bipolar I disorder and responded to divalproex $125 \mathrm{mg}$ bid and risperidone $0.25 \mathrm{mg}$ bid over one month in hospital. Her Mini-Mental State Examination (MMSE) score on the day of admission was $24 / 30$, with points lost on recall $(0 / 3)$ and orientation (7/10). Once stabilized one month later, her Montreal Cognitive Assessment (MoCA) score was 16/30 showing persistent cognitive impairment, with points lost for visuospatial/executive function (2/5), attention (2/6), delayed recall (1/5), abstraction (0/2), orientation (5/6) and language fluency (0/1). Subsequently, she was followed as an outpatient by the geriatric mental health service. She remained fairly stable on divalproex $125 \mathrm{mg}$ bid but declined cognitively and her MoCA score was $19 / 30$ in 2010. Thereafter, she started deteriorating further and had a MoCA score of 9/30 in 2012 associated with impairments in her activities of daily living, attention, language, and executive function on functional assessments. She was diagnosed with dementia and started on galantamine at $8 \mathrm{mg}$ daily.

The family soon noted that the patient was demonstrating unusual elation, expansiveness, decreased need for sleep, pressured speech, distractibility, psychomotor agitation that included walking 8-10 miles daily, hypersexuality, and incessant counting and dancing. Her galantamine as well as her psychiatric medications were discontinued after two weeks, but over the next week she continued to deteriorate and presented to the

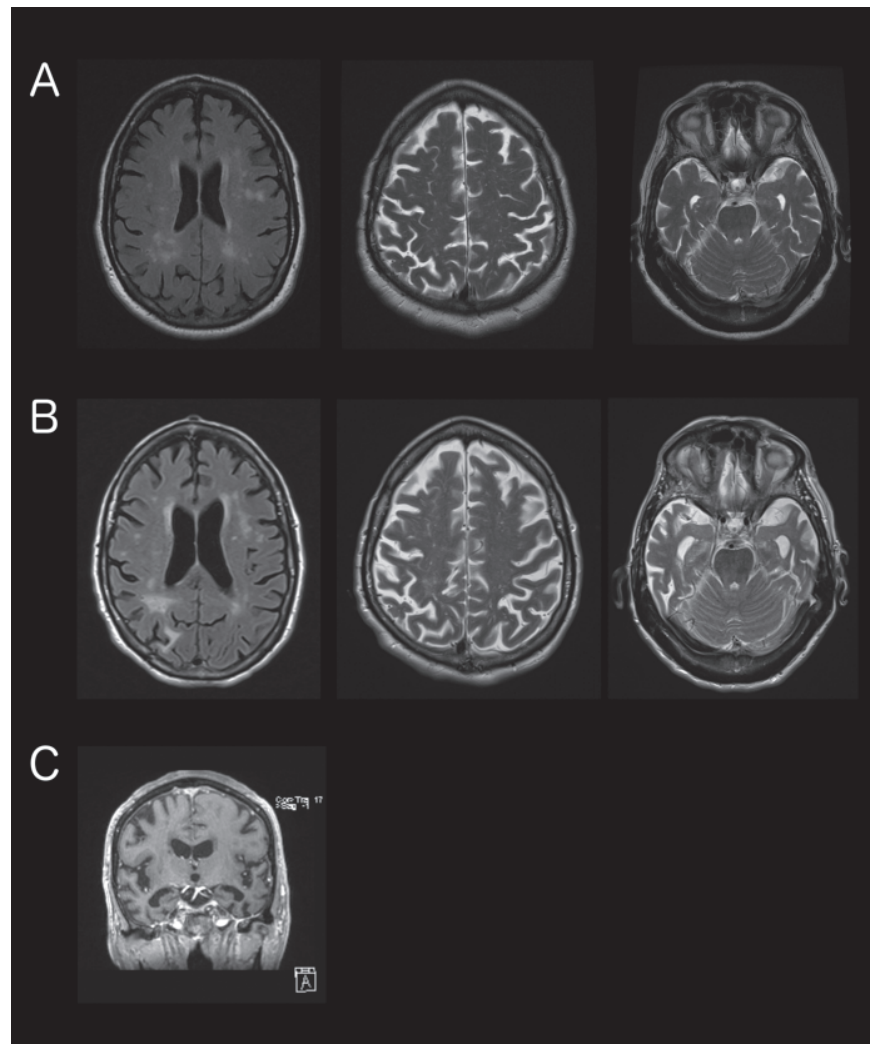

Figure 1: Panel A shows axial MRI scans of the patient from September 2007 of the lower frontoparietal (FLAIR; left), higher frontoparietal (T2 weighted; middle), and temporal regions (T2 weighted; right). These images demonstrate moderate white matter disease (WMD) with mild temporal and parietal lobe atrophy. Panel B shows axial MRI scans of the corresponding regions from October 2012. These images demonstrate significantly worse WMD and temporal lobe atrophy. Panel $C$ is a coronal gadolinium-enhanced $T 1$ weighted image showing bilateral hippocampal atrophy (October 2012).

From the Department of Neurology (HJ), University of Toronto, Toronto, Ontario; Department of Clinical Neurosciences (AG, ZI), Departments of Radiology and Surgery (JL), Department of Psychiatry (RL, ZI), Hotchkiss Brain Institute (ZI), University of Calgary, Calgary, Alberta, Canada.

Received July 19, 2013. Final Revisions Submitted October 2, 2013. Correspondence to: Zahinoor Ismail, Departments of Psychiatry and Clinical Neurosciences, Hotchkiss Brain Institute, University of Calgary, Calgary, Alberta, Canada.Email: zahinoor@gmail.com. 


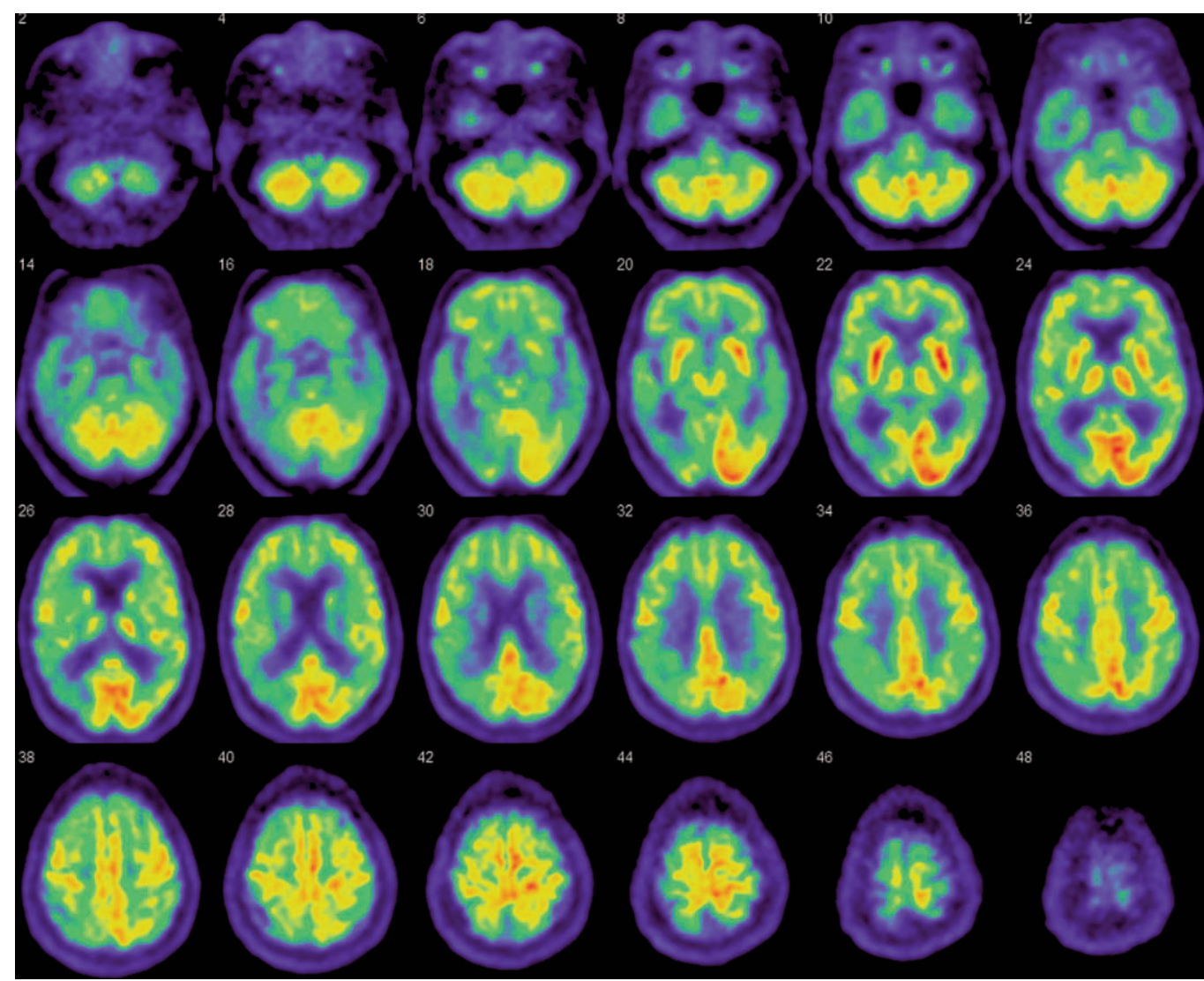

Figure 2: Positron Emission Tomography demonstrating parietal, temporal and patchy frontal hypometabolism.

hospital where she was recognized to be frankly manic. An extensive work-up for medical causes of mania or delirium, including hemoglobin, electrolytes, functional tests of the liver, kidneys and thyroid, drug and toxicology screens, vasculitis screen, ceruloplasmin, human immunodeficiency virus (HIV) testing, syphilis antibody screen, as well as computed tomography scans of the chest, abdomen, and pelvis for neoplasms, was unremarkable. Magnetic resonance imaging (MRI) of her brain in 2007 had demonstrated mild temporal and parietal atrophy with moderate leukoaraiosis. Five years later, MRI demonstrated significantly worse temporal lobe atrophy, including hippocampal atrophy, as well as some worsening of leukoaraiosis (see Figure 1). A positron emission tomography scan (FDG-PET) demonstrated temporal and parietal lobe hypometabolism $(\mathrm{R}>\mathrm{L})$, and patchy frontal hypometabolism, consistent with progressive AD likely mixed with a vascular component (see Figure 2). She was started on divalproex and risperidone and her mania resolved. Three weeks later she was euthymic with sleep, speech, and behaviour back to baseline. At discharge, her MMSE score was 15/30 with points lost for orientation $(6 / 10)$, registration $(0 / 3)$, attention $(2 / 5)$, recall $(1 / 3)$, language 6/8) and construction (0/1). Her MoCA score was 7/30 losing points for visuospatial/executive function (1/5), language $(1 / 5)$, attention $(0 / 2)$, abstraction $(0 / 2)$, recall $(0 / 5)$ and orientation $(3 / 6)$

\section{Discussion}

We present a case of CHEI-associated mania in an elderly patient with probable $\mathrm{AD}$, and a bipolar diathesis that was unmasked at the index presentation of cognitive impairment five years previously. Eight similar cases have been previously reported with onset of manic symptoms soon after CHEI initiation. The first two cases were published in 1998, associating donepezil use with mania onset in one patient previously diagnosed with major depressive disorder, and in another with bipolar I (See summary in ${ }^{1}$ ). Three more cases were reported associating donepezil use with mania or hypomania in patients with previous depression ${ }^{3}$, bipolar, or delusional disorders ${ }^{1}$. There was one report of a patient with no psychiatric history developing hypomania after donepezil use ${ }^{4}$. Finally, two cases were reported of patients with bipolar I developing mania after initiation of rivastigmine and galantamine ${ }^{2}$.

On the other hand, there have also been reports of a positive impact of CHEIs on bipolar spectrum disorders, though it remains controversial. A case-series in 1999 reported marked improvement with add-on donepezil in 6 of 11 patients with treatment-resistant bipolar disorder ${ }^{5}$.

This uncertainty in the effect of CHEI on bipolar diathesis may reflect our incomplete understanding of (1) the neurophysiological effects of CHEIs and (2) the role of the central 
cholinergic system, in the different settings of bipolar spectrum disorders, dementia, or combined bipolar diathesis and dementia. With respect to (1), galantamine is also a ligand of neuronal nicotinic receptors, which could enhance the function of other neurotransmitters including dopamine, noradrenaline, serotonin and glutamate ${ }^{5}$. It is also possible that either the underlying bipolar diathesis itself, or the mood-stabilizers used for treatment in these patients, may be directly contributing to neuronal loss and therefore predisposing them to CHEI-associated mania. With respect to (2), cognitive impairment caused by underlying cholinergic deficits may make these patients vulnerable to mania and other NPS. Thus, ongoing neurodegeneration, as evidenced by progressive MRI changes may have contributed to the patient's increased likelihood of having neuropsychiatric symptoms such as mania. It is possible that this increased likelihood or diathesis was then exposed by addition of galantamine. The fact that her mania resolved completely after cessation of galantamine supports the notion that it was the most likely causative factor in this episode of mania.

Ultimately, seen in the context of other reports of CHEIassociated mania, our case report suggests that use of CHEIs may need to be more cautiously implemented in cognitively impaired elderly patients with apparent concomitant psychopathology.

\section{REFERENCES}

1. Benazzi F. Mania associated with donepezil. J Psychiatry Neurosci. 1999;24(5):468-9.

2. Ehrt U, Fritze F, Aarsland D. Mania after administration of cholinesterase inhibitors in patients with dementia and comorbid bipolar disorder: two case reports. J Clin Psychopharmacol. 2011;31(2):254-6.

3. Rao V, Ovitt L, Robbins B. Donepezil induced hypomania. J Neuropsychiatry Clin Neurosci. 2008;20(1):107.

4. Collins C, Copeland B, Croucher M. Bipolar affective disorder, type II, apparently precipitated by donepezil. Int Psychogeriatr. 2011;23(3):503-4.

5. Burt T, Sachs GS, Demopulos C. Donezepil in treatment-resistant bipolar disorder. Biol Psychiatry. 1999;45(8):959-64. 